\title{
Reconstruction of the transformer theory
}

\author{
Mansur Shakirov ${ }^{1, *}$ \\ ${ }^{1}$ Peter the Great St. Petersburg Polytechnic University, 29 Politekhnicheskaya street, Saint- \\ Petersburg, 195251, Russian Federation
}

\begin{abstract}
The fallacy of the traditional transformer theory is illustrated. The fundamentals of the new theory, beginning from principle of the transformer action, motion of electromagnetic field in its window, new equivalent circuit and vector diagrams are discussed. More correct decision for the sudden short-circuit in real transformers are presented.
\end{abstract}

\section{Introduction}

The traditional transformer theory is based on the artificial notion of the so-called "magnetizing current $i_{0}=\left(w_{1} i_{1}-w_{2} i_{2}\right) / w_{1}$ ", which allegedly is responsible for producing socalled "mutual magnetic flux $\Phi_{0}$ in core". The latter is associated with the fabrications of the "leakage flux" for each winding and the "leakage inductance of each winding $X_{L 1}, X_{L 2}$ " [14]. In [5-10] it is shown also, as gradually during a 100 year, objecting grew against this theory. To overcome that crisis it is necessary to create such equivalent circuits, where each element of the core, the windings and the window would have the concrete mapping [1114]. The new theory description should be phased (step by step): at first, for the simplest cases in order to ensure the availability of understanding of the real principle of the transformer action, the transfer of energy in it, the procedure for constructing equivalent circuits. Some of these problems are being tackled for the first time in the present work.

\section{Methods}

\subsection{Model for the explanation of the transformer operation}

In describing the principle of operation of any device should be considered its idealized model. Such idealized model for the transformer is shown in Fig. 1. First it is natural to consider the case of infinitely thin coils. For an infinite permeability magnetic material $w_{1} i_{1}=$ $w_{2} i_{2}$.

\footnotetext{
${ }^{*}$ Corresponding author: manshak@mail.ru
} 
a)

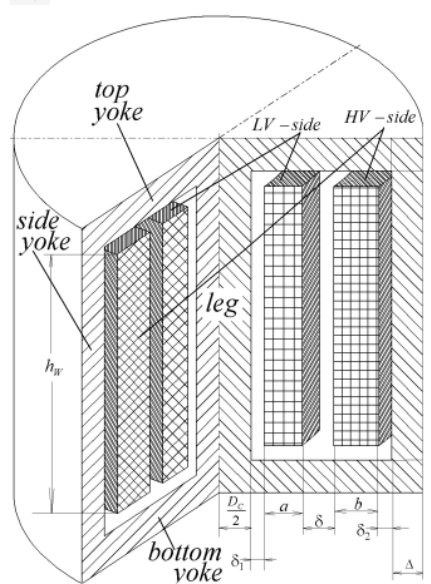

b)

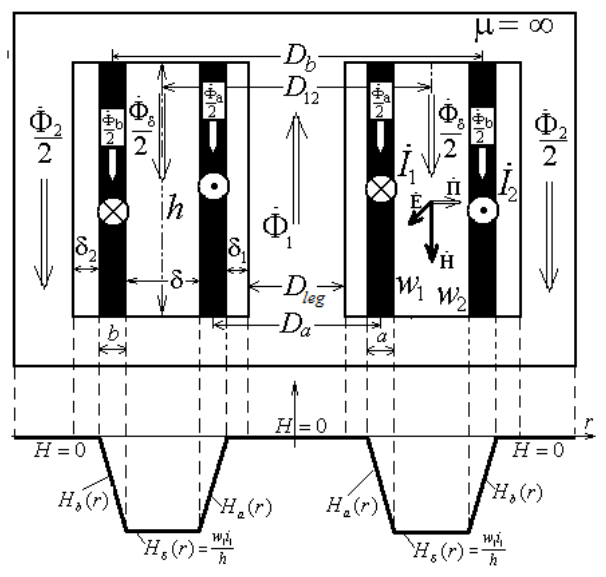

Fig. 1. Shell-type transformer $(a)$ and its cross section $(b)$ for idealized case $(\mu=\infty)$

\subsection{So how does the transformer work?}

The new understanding includes the following provisions:

1. In fact, the pair of windings is a system, that is intended to create a difference of magnetic potentials (correctly, magnetic tension that is equal to $u^{\mathrm{M}}=w_{1} i_{1}=w_{2} i_{2}$ ) in the window space. Under the influence of this potential difference, operating magnetic flux $\Phi_{\delta}$. It is the common flux in window and it can not be divided between the windings.

2. The window is not a leakage zone. This is actually a working area for energy transfer from primary winding to secondary with the Poynting vector $\dot{\Pi}(r)$ (Fig. 1b).

3. It should be borne in mind that the phase angles of fluxes ( $\Phi_{1}, \Phi_{2}$ and $\Phi_{\delta}$ ) are not the same:

$$
\dot{\Phi}_{1}=\dot{\Phi}_{\delta}+\dot{\Phi}_{2} .
$$

It is easy to show that the existence of the angle $\theta$ between $\dot{\Phi}_{1}$ and $\dot{\Phi}_{2}$ is the condition for the power transfer from the primary to the secondary ones $[15,16]$.

\subsection{Transformer with the thin windings}

The equivalent circuit of an idealized transformer should not contain any shunt branches (Fig.

2). It is obvious that for thin windings angle $\theta_{\psi}=\theta$ :

$$
\operatorname{tg} \theta=\frac{x_{\text {sh }}\left(R_{2}^{\prime}+R_{\text {load }}^{\prime}\right)}{\left(R_{2}^{\prime}+R_{\text {load }}^{\prime}\right)^{2}+X_{\text {load }}^{\prime}\left(x_{\text {sh }}+X_{\text {load }}^{\prime}\right)} .
$$


a)

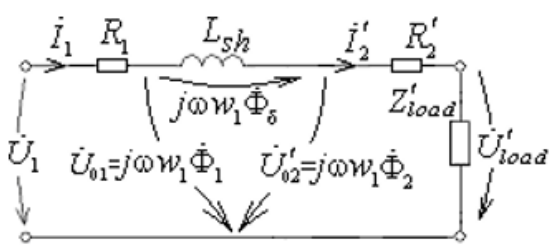

b)

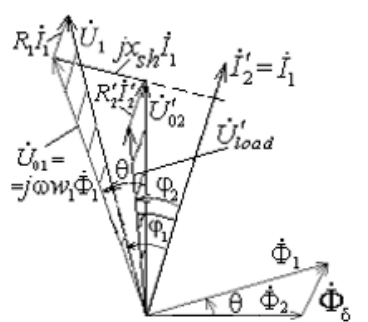

Fig. 2. A new view at an equivalent circuit of the idealized transformer $(a)$ and its vector diagram with a triangle magnetic fluxes $(b)$ in the case of thin windings

From the equivalent circuit we can obtain equations and construct the curves of fluxes and angle $\theta$ depending on the load current, as it is shown in Fig. 3a, where $\Phi_{0}$ is the

a)

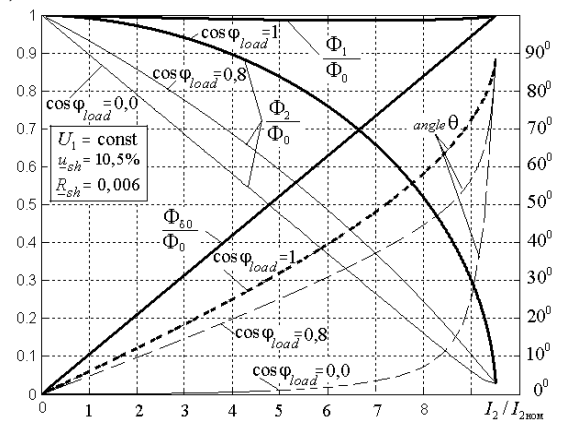

b)

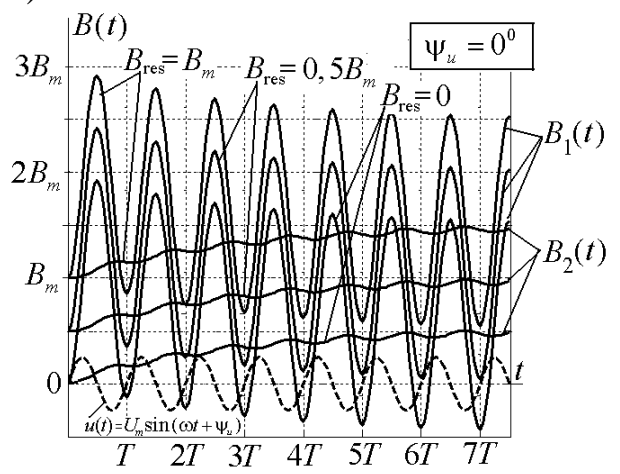

Fig. 3. Steady-state regimes in the idealized transformer with thin windings $(a)$ and magnetic flux density in its leg and side yoke at sudden short-circuit on HV-side (b)

magnetic flux in the leg in no load condition. It is seen that while the flux $\Phi_{1}$ in the leg is almost unchanged, the flux $\Phi_{2}$ in the side yoke drops almost to zero (more precisely to ( $\left.\left.R_{2}^{\prime} / z_{s h}\right) \Phi 0\right)$. Here $z_{s h}$ is the modulus of short-circuit complex impedance:

$$
Z_{s h}=\left(R_{1}+R_{2}^{\prime}\right)+j \omega L_{s h}=R_{s h}+j x_{s h} .
$$

The traditional view on mutual flux is refuted by the formula for the active power transferring from primary winding to secondary one:

$$
P \equiv \Phi_{1} \Phi_{2} \sin \theta
$$

In a nominal regime angle $\theta$ between fluxes equals $6^{0}-8^{0}$, but in short-circuit it approaches (in the case of thin windings!) almost $90^{\circ}$. Fig. $3 \mathrm{~b}$ shows the curves of the magnetic flux density for the most dangerous case:

1. Sudden short-circuit leads to the introduction of permanent magnetic flux density:

$$
\Delta B_{\text {const }}=\frac{B_{m}}{2} \cos \psi_{u}
$$

2. The characters of the magnetic flux density in the leg and the side yoke are significantly different.

3. Even in the absence of residual magnetic flux density $\left(B_{\mathrm{res}}=0\right)$ the flux in the leg greatly exceeds the value of the magnetic flux density of idling. The greatest value of it is: 


$$
B_{\text {greatest }}=\left.B_{1}(t)\right|_{\substack{\psi_{u}=0 \\ t=T / 2}} \approx B_{m}\left(1,5+0,5 \cdot e^{-\frac{T}{2 \tau_{s h}}}\right)+B_{\text {res }} ; \quad \tau_{s h}=L_{s h} / R_{s h} .
$$

4. The curves indicate that until the latest time the calculated peak of short-circuit current appears to be rather low, as in the traditional theory the core saturation during a shortcircuit is not taken into account.

\subsection{Transformer with the finite thickness windings}

In corresponding equivalent circuit (Fig. 4):

$$
L_{a} \approx \frac{\mu_{0} \pi w_{1}^{2}}{h} \frac{a D_{a}}{3} ; \quad L_{\delta}=\frac{\mu_{0} \pi w_{1}^{2}}{h} \delta D_{12} ; \quad L_{b}^{\prime} \approx \frac{\mu_{0} \pi w_{1}^{2}}{h} \frac{b D_{b}}{3} .
$$

In the new theory the parameters (7) are treated as internal (not leakage) series inductances of a transformer. The general expression for angle $\theta$ is more complicated. For example in case of a resistive load ( $\left.Z_{\text {load }}=R_{\text {load }}\right)$ it has the form:

$$
\left.\operatorname{tg} \theta\right|_{Z_{\text {load }}=R_{\text {load }}}=\frac{\left(x_{\text {sh }}+\frac{\omega\left(L_{a}+L_{b}^{\prime}\right)}{2}\right)\left(R_{\text {load }}^{\prime}+R_{2}^{\prime}\right)}{\left(R_{\text {load }}^{\prime}+R_{2}^{\prime}\right)^{2}-\frac{\omega L_{b}^{\prime}}{2}\left(x_{\text {sh }}+\frac{\omega L_{a}}{2}\right)} .
$$

From this expression it follows that in the short-circuit mode for this angle we can write:

$$
\theta_{s h} \approx\left(\pi-\operatorname{arctg} \frac{2 R_{2}^{\prime}}{\omega L_{b}^{\prime}}\right) \rightarrow \pi .
$$

The expression (9) shows that during the short circuit the flux $\Phi_{2}$ is directed almost upstream $\Phi_{1}$. So it is called the anti-flux in the short-circuit mode. The influence of finite thickness of windings is seen from comparison between curves on Fig. 3 and Fig. 5.

a)

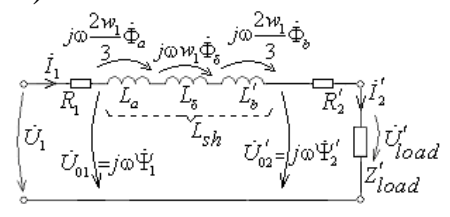

b)

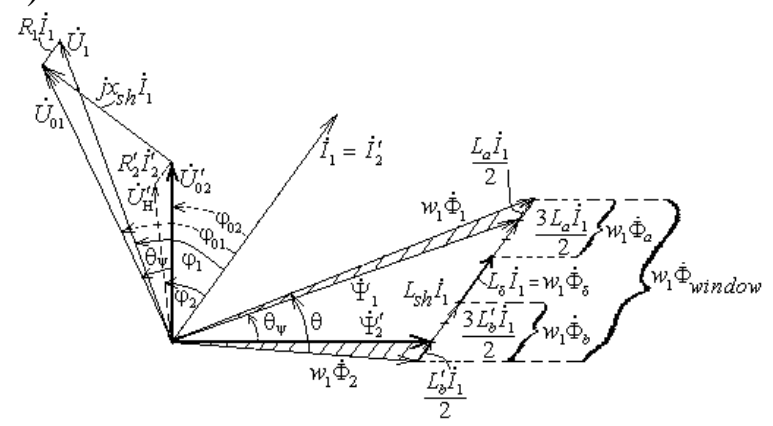

Fig. 4. The new approach to analyzing an equivalent circuit of the idealized transformer $(a)$ and its new vector diagram with a triangle of fluxes $(b)$ in the case of windings of finite thickness 
a)

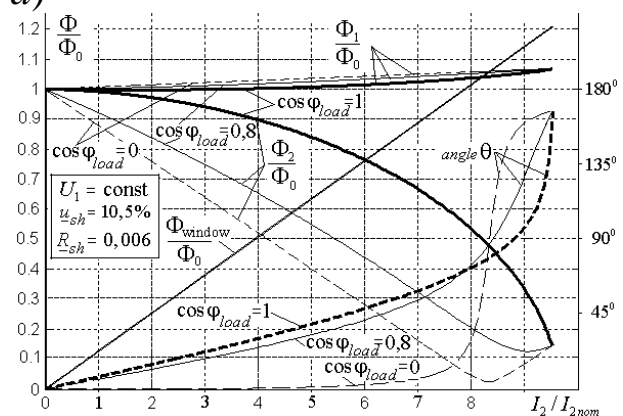

b)

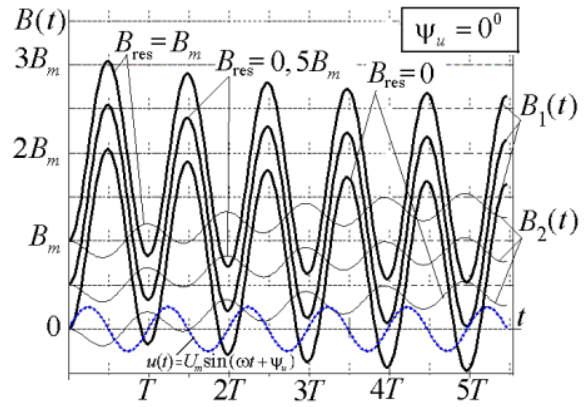

Fig. 5. Steady-state regimes in the transformer with windings of finite thickness $(a)$ and magnetic flux density in its leg and side yoke after sudden short-circuit on $\mathrm{HV}$-side (b)

The curves (Fig. 5) were obtained from equivalent circuit (Fig. 4a). The formula for the evaluation of anti-flux has the form:

$$
k_{2}^{s h}=\left.\frac{\Phi_{2}}{\Phi_{0}}\right|_{Z_{\text {loud }}=0} \approx \frac{L_{b}^{\prime}}{2 L_{\mathrm{K} 3}}=\frac{b\left(D_{12}+\delta+b\right)}{6\left[D_{12}\left(\delta+\frac{a+b}{3}\right)+\frac{b-a}{3}\left(\delta+\frac{(b+a)}{2}\right)\right]} \approx \frac{b}{2 a+6 \delta+2 b} .
$$

The curves (Fig. 5a) also show that the flux $\Phi_{1}$ in shot circuit exceeds the flux of idling $\Phi_{0}$. So it is called super-flux in short circuit mode:

$$
k_{1}^{s h}=\left.\frac{\Phi_{1}}{\Phi_{0}}\right|_{Z_{\text {bud }}=0} \approx 1+\frac{L_{a}}{2 L_{\mathrm{K} 3}}=1+\frac{a\left(D_{12}-\delta-a\right)}{6\left[D_{12}\left(\delta+\frac{a+b}{3}\right)+\frac{b-a}{3}\left(\delta+\frac{(b+a)}{2}\right)\right]} \approx 1+\frac{a}{2 a+6 \delta+2 b}>1 .
$$

The equations (10) and (11) show a gross mistake of the traditional theory according to which in short-circuit mode $\Phi_{1}=\Phi_{2}=\Phi_{0} / 2$. The curve for flux in the gap with the width of $\delta$ shows that

$$
k_{\delta}^{s h}=\left.\frac{\Phi_{\delta}}{\Phi_{0}}\right|_{Z_{\text {load }}=0}=\left(\frac{\Phi_{1}}{\Phi_{0}}+\frac{\Phi_{2}}{\Phi_{0}}\right)_{Z_{\text {load }}=0} \approx 1+\frac{L_{a}+L_{b}^{\prime}}{2 L_{\mathrm{K} 3}} \approx 1+\frac{a+b}{2 a+6 \delta+2 b}>1 .
$$

\subsection{Power flow in idealized transformer}

As should be evident from the some textbooks, the complex power flows through the gap with the width $\delta$ (Fig.1) via the Poynting vector $\dot{\Pi}_{\delta}(r)$ :

$$
\dot{S}_{\delta}(r)=P_{\delta}(r)+j Q_{\delta}(r)=2 \pi r h \cdot \dot{\Pi}_{\delta}(r) ; \quad \dot{\Pi}_{\delta}(r)=\dot{E}_{\delta}(r) H_{\delta}^{*}(r),
$$

and is directed from the primary towards the secondary winding. The active power of an idealized transformer flows through the $\delta$-gap without loss:

$$
2 \pi r h \cdot \operatorname{Re}\left(\dot{\Pi}_{\delta}(r)\right)=\text { const }
$$

\subsubsection{E-field}

The law of $E$-field distribution is determined by the law of electromagnetic induction: 


$$
\oint E(r) d l=2 \pi r E(r)=\frac{d \Phi(r)}{d t} ; \quad \dot{E}(r)=\frac{j \omega \Phi(r)}{2 \pi r} .
$$

Found with these formulas the distributions of the modulus and phase of $E(r)$ for different types of rated load and for the short-circuit mode are shown in Fig. 6. Curves in Fig.6a

a)

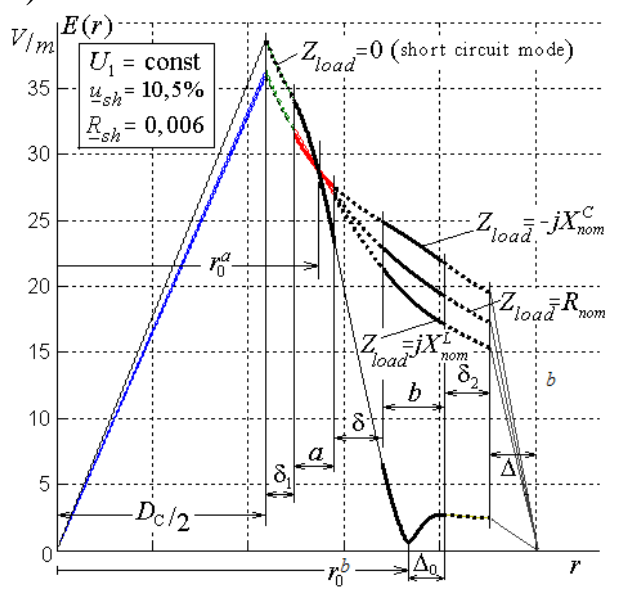

b)

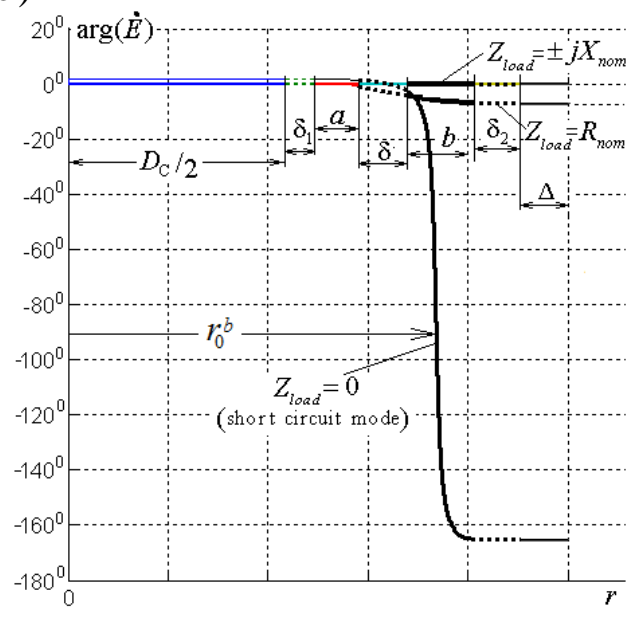

Fig. 6. The curves of modulus $(a)$ and phase $(b)$ of the electric field in an idealized transformer with the rated active, inductive and capacitive loads in comparison with the curves of short-circuit mode

illustrate an important scientific discovery for transformer - the existence of an $E$ invariant cylindrical surface inside the primary $a$-winding, named so because the electric field strength at its points does not depend on the load. The radius of this surface is

$$
r_{0}^{a} \approx r_{1}^{a}+\frac{a}{\sqrt{3}} ; \quad r_{1}^{a}=\frac{D_{a}}{2}-a .
$$

\subsubsection{H-field}

The law of $H$-field distribution is given in any textbook, it is represented in Fig.1.

\subsubsection{The Poynting vector}

The Poynting vector directions on the inner and outer sides of the short-circuited winding are almost mutually opposite and there is a cylindrical surface of radius

$$
r_{0}^{b} \approx r_{2}^{b}-\frac{b}{\sqrt{3}} ; \quad r_{2}^{b}=\frac{D_{b}}{2}+b
$$

where the Poynting vector is close to zero (when the imaginary component is exactly zero).

\section{Results and discussion}

\subsection{Visualization of the super- and anti-fluxes}

In order to observe the field in the core it is necessary to use a finite steel permeability 


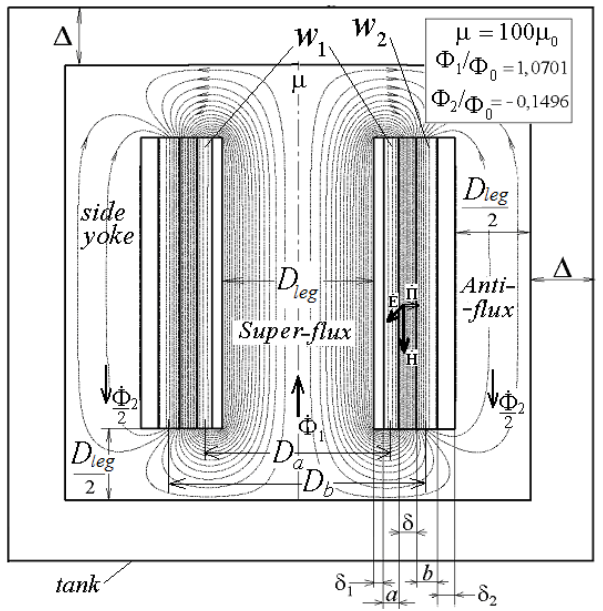

Fig. 7. Super- and anti-fluxes in short-circuit mode of a linear transformer $\left(\Phi_{1}-\right.$ flux in leg, $\Phi_{2}-$ flux in side yokes, $\Phi_{0}$ - core flux in idling regime)

$\mu=(10 \div 1000) \mu_{0}$. Field picture in Fig. 7 was obtained assuming that the active resistance of the windings $R_{1}=R_{2}=0$. Obviously super- and anti-fluxes (and a new theory in General) should be confirmed by such experiments that can easily be reproduced in any university student laboratory. At the Peter the Great St. Petersburg Polytechnic University four such units were created [17-20].

\subsection{New 4T-shaped equivalent circuit}

According to the above it follows that now the requirement for the equivalent circuits to correctly display the processes in normal and abnormal modes of a transformer means that they must feel its super- and anti-fluxes in short-circuit. Meanwhile, it gradually became clear about the exceptional role of the Poynting vector in the understanding of the operating principle of a transformer. In this regard, in [21-23] an attempt was made to direct construction of the equivalent circuits on the basis of the movement of electromagnetic energy in the transformer. In the beginning was built the exact model for an idealized transformer $(\mu=\infty)$ with displaying all of its magnetic fluxes. After that from it was derived heuristic equivalent circuit of a real transformer by adding four transverse branches (Fig. 8), showing the magnetic losses in the saturated steel of leg, side yoke, in butt yokes on $a$-side and on $b$-side. From it it is possible to obtain well-known T-and $\pi$-shaped equivalent circuits and more correctly assess their role and importance in the modeling of electric power networks and systems.

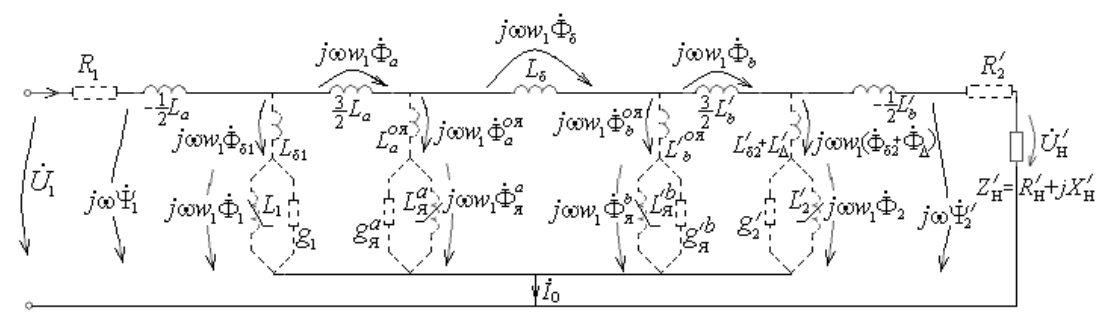

Fig. 8. 4T-shaped equivalent circuit of a transformer 


\section{Conclusions}

The End of the traditional transformer theory was predetermined in connection by the huge number of researchers, which had criticized $\mathrm{T}$ - and $\Pi$ - equivalent circuits long ago. The presented theory is revolutionary because it does not develop or improve an old theory, but denies it and declares it to be wrong. The totality of the traditional basic concepts completely replaced with the new concepts. Correctness of the new 4T-shaped equivalent circuits is confirmed by their "ability to feel" super- and anti-fluxes in the different parts of the core while short-circuit. The calculation of the shock short-circuit current should be carried out taking magnetic permeability not equal to infinite, as in the old theory, but rather as $\mu_{\text {steel }}=\mu_{0}$. The new theory permits to solve all problems within the Poynting vector and on this basis to present a more realistic conception of the transformer work.

\section{References}

1. F. Leon, P. Gomez, Martinez-Velasco, M. Rioual, Transformers in Power System Transients: Parameter Determination. FL: CRC, 177-250 (2009)

2. F.Leon, A.Farazmand, L.Joseph, IEEE Trans. Power Delivery, 27(4), 2390-2397 (2012)

3. C.Steinmetz, Theory and Calculation of Alternating Current Phenomena. McGraw-Hill Book Company. NY (1897)

4. A.Boyajian, AIEE Trans. XLIV, 805-810 (1925).

5. E.Markvardt, Electromagnetical Calculatings of Transformers. Moscow. United Scientifically -Technical Publ. Hause (1938)

6. P. Penchev, V'erkhu razseivaneto vtransformatorite. Sofija, Technology (1969)

7. E.Cherry, Proc. of the Physical Society., B 62, 101-111 (1949)

8. L.Blume, A.Boyajian,G. Gamilly, T.Lenox, S.Minnec, M.Montsinger, Transformer Engineering: A treatise on the Theory, Operation and Application of Transformer. New York: Wiley (1951)

9. G.Slemon, Proc. Inst. Elect. Eng., IV (100), 129-143 (1953)

10.L.Leites, A.Pinsov, Skhemy zameshcheniy mnogoobmotochnikh transformatorov, Moscow. Publ. Energiya (1974)

11. M.Shakirov, Electrichestvo, (11), 34-45 (2003)

12. M.Shakirov, Electrichestvo, (11), 15-27 (2005)

13. G.Aleksandrov, M.Shakirov, Proc. of the Russian Academy of Sciences. Energetika, (4), 70-90 (2005)

14. M.Shakirov, Electrichestvo, (5), 19-36 (2010)

15. M.Shakirov, Theoretical electrical engineering. Tensors. Electrodynamics. General relativity. SPbGPU, St. Petersburg, Russia (2011)

16. M.Shakirov, St. Petersburg State Polytechnic University Journal of Engineering Science and Technology, 154(3-2), 85-110 (2012)

17. M.Shakirov, U.Varlamov, D.Liyung, St. Petersburg State Polytechnic University Journal of Engineering Science and Technology, 95(1), 107-120 (2010)

18. M. Shakirov, V.Andrushchuk, D. Liyung, Electrichestvo, (3), 55-63 (2010)

19. M. Shakirov, V.Andrushchuk, U.Varlamov, Proc. of OIPE. Sept. 19-21. Ghent, Belgium, 208-209 (2012) 
20. M. Shakirov, Intern. Symp. on Applied Electromagnetics and Mechanics (ISEM 2013), Laval Univ. in Quebec City, Canada. Abstract Book of the 16-th edition of the ISEM, 339-340 (2013).

21. M.Shakirov, Electrichestvo, (9), 52-59 ( 2014)

22. M.Shakirov, Electrichestvo, (10), 53-65 ( 2014)

23. M.Shakirov, Electrichestvo, (3), 37-49 ( 2017) 